\title{
Test of steady-state fluctuation theorem in turbulent Rayleigh-Bénard convection
}

\author{
X.-D. Shang, ${ }^{1,2}$ P. Tong, ${ }^{1,3}$ and K.-Q. Xia ${ }^{2}$ \\ ${ }^{1}$ Department of Physics, Oklahoma State University, Stillwater, Oklahoma 74078, USA \\ ${ }^{2}$ Department of Physics, the Chinese University of Hong Kong, Shatin, Hong Kong \\ ${ }^{3}$ Department of Physics, Hong Kong University of Science and Technology, Clear Water Bay, Kowloon, Hong Kong
}

(Received 19 December 2004; published 29 July 2005)

\begin{abstract}
The local entropy production rate $\sigma(\mathbf{r}, t)$ in turbulent thermal convection is obtained from simultaneous velocity and temperature measurements in an aspect-ratio-one cell filled with water. The statistical properties of the time-averaged $\sigma(\mathbf{r}, t)$ are analyzed and the results are compared with the predictions of the steady-state fluctuation theorem (SSFT) of Gallavotti and Cohen. The experiment reveals that the SSFT can indeed be extended to the local variables, but further development is needed in order to incorporate the common dynamic complexities of far-from-equilibrium systems into the theory.
\end{abstract}

DOI: 10.1103/PhysRevE.72.015301

PACS number(s): 47.27.Te, 05.40.-a, 05.70.Ln

While much is known about fluctuations at equilibrium, our current understanding of fluctuations in systems far from equilibrium is rather limited [1]. Large nonequilibrium fluctuations may occur in macroscopic systems subject to an external driving force or in microscopic systems, such as nanoscale devices and biomolecules including DNA and proteins, in contact with a thermal bath of temperature $T$. For such small systems with relevant energies comparable to the thermal energy $k_{B} T$, one often observes large deviations from the average behavior. Recently, a set of closely related fluctuation theorems [2] were proposed to describe nonequilibrium fluctuations of the time-averaged entropy production rate $\sigma_{\tau} \equiv(1 / \tau) \int_{t}^{t+\tau} \sigma\left(t^{\prime}\right) d t^{\prime}$. While the range of applicability and the statistical mechanical ensembles used differ, these theorems have the same general form

$$
\frac{P(+\sigma)}{P(-\sigma)} \simeq e^{\sigma \tau},
$$

where $+\sigma$ and $-\sigma$ are equal but opposite values of $\sigma_{\tau}$, and $P(+\sigma)$ and $P(-\sigma)$ are the corresponding probability density functions. The fluctuation theorems (FTs) were originally derived for global variables and later it was conjectured $[2,3]$ that FTs may also apply to the time-averaged local entropy production rate $\sigma_{\tau}(\mathbf{r}) \equiv(1 / \tau) \int_{t}^{t+\tau} \sigma\left(\mathbf{r}, t^{\prime}\right) d t^{\prime}$.

In this Rapid Communication, we report an experimental study on fluctuations of local heat transport in a far-fromequilibrium system of turbulent Rayleigh-Bénard convection, in which one applies a vertical temperature difference $\Delta T$ across a horizontal layer of fluid of thickness $H$, so that heat is transferred vertically from the lower warm surface to the upper cold surface. When the Rayleigh number Ra $\left[=\alpha g \Delta T H^{3} /(\nu \kappa)\right.$, where $g$ is the gravitational acceleration, and $\alpha, \nu$, and $\kappa$ are, respectively, the thermal expansion coefficient, the kinematic viscosity, and the thermal diffusivity of the fluid] is large enough $\left(\mathrm{Ra} \gtrsim 10^{8}\right)$, the bulk fluid becomes turbulent and heat is transported predominantly by convection $[4,5]$. In the experiment, we simultaneously measure the local velocity $\mathbf{v}(\mathbf{r}, t)$ and the local temperature $T(\mathbf{r}, t)$, from which we obtain the local convective heat flux [6]

$$
\mathbf{j}(\mathbf{r}, t)=\frac{\mathbf{v}(\mathbf{r}, t) \delta T_{0}(\mathbf{r}, t)}{\kappa \Delta T / H},
$$

where $\delta T_{0}(\mathbf{r}, t)$ is defined as $\delta T_{0}(\mathbf{r}, t)=T(\mathbf{r}, t)-T_{0}$ with $T_{0}$ being the mean temperature of the bulk fluid. In Eq. (2), $\mathbf{j}(\mathbf{r}, t)$ has been normalized by the conductive heat flux $j_{c}$ $=k \Delta T / H$, where $k$ is the thermal conductivity of the fluid. The measured vertical heat flux $j_{z}(\mathbf{r}, t)$ is directly related to $\sigma(\mathbf{r}, t)$ through the equation [7] $\sigma(\mathbf{r}, t)=v_{0} j_{c} j_{z}(\mathbf{r}, t) \nabla_{z}(1 / T)$, where $v_{0}$ is a small volume over which the local velocity and temperature measurements are averaged, and $\nabla_{z}(1 / T)$ is the thermodynamic force (in the $z$ direction) that drives $j_{z}(\mathbf{r}, t)$. To test the steady-state FT (SSFT) of Gallavotti and Cohen [8] for the local variables, we compute a running average of $j_{z}(\mathbf{r}, t)$ and obtain the time series $J_{\tau}(\mathbf{r}, t)$ $\equiv(1 / \tau) \int_{t}^{t+\tau} j_{z}\left(\mathbf{r}, t^{\prime}\right) d t^{\prime}$. With $J_{\tau}(\mathbf{r}, t)$ being identified as the fluctuating variable of interest, Eq. (1) becomes

$$
\frac{P\left(+J_{\tau}\right)}{P\left(-J_{\tau}\right)} \simeq e^{\left[v_{0} j_{c} \nabla_{z}(1 / T) / k_{B}\right] J_{\tau} \tau},
$$

where $v_{0} j_{c} \nabla_{z}(1 / T) / k_{B}$ is a numerical factor to be determined below.

In addition to the experimental studies of the SSFT for global variables [9], several experiments have been carried out to test the applicability of the SSFT to local variables, including fluctuations of the power injection required for sustaining the dynamics in a vertically agitated granular gas [10], temperature fluctuations in turbulent convection [11], and fluctuations of the force exerted on a small sensor plate placed in a turbulent flow between two counterrotating disks and in a wind tunnel [12]. These experiments provided important insights into the nature of nonequilibrium fluctuations but also revealed some uncertainties involved in testing the SSFT. For example, the experiments by Ciliberto et al. concerned the temperature and force fluctuations to which the SSFT does not apply. As shown in Eq. (2), $j_{z}(\mathbf{r}, t)$ contains a fluctuating velocity factor and thus obeys different statistics from that of $\delta T_{0}$ [6]. The measured force is an integral of the local pressure over the sensor plate, which bears no direct relation with $\sigma(\mathbf{r}, t)$. Feitosa and Menon have 
pointed out [10] that the conditions of time reversibility or detailed balance essential for the proof of the SSFT do not apply to the granular flows. Clearly, direct measurements of $\sigma(\mathbf{r}, t)$ are needed in order to further explore the applicability of the SSFT to a variety of far-from-equilibrium systems, whose governing equations of motion satisfy the conditions required by the SSFT.

Our experiment is conducted in an upright cylindrical cell filled with water. The aspect ratio of the cell is unity and the cell height is $H=20.5 \mathrm{~cm}$. Details about the apparatus and the experimental method have been described elsewhere [6], and here we mention only some key points. Local velocity measurements are performed using a laser Doppler velocimetry (LDV) system [13], which can simultaneously measure two components of $\mathbf{v}(\mathbf{r}, t)$. Temperature measurements are simultaneously taken using a multichannel LDV interface module for synchronization of the data acquisition. A small movable thermistor of $0.2 \mathrm{~mm}$ diam, $15 \mathrm{~ms}$ response time, and $20 \mathrm{mK} / \Omega$ temperature sensitivity is used to measure $T(\mathbf{r}, t)$. The sampling rate of the measurements is $10-15 \mathrm{~Hz}$, which is 3-10 times (depending on the value of $\mathrm{Ra}$ ) larger than the cutoff frequency of the velocity power spectrum. Typically, we take seven hour-long time series data $(\sim 5$ $\times 10^{5}$ data points) at each location, ensuring that the statistical average of the flow properties is adequate. The measurements of $\mathbf{v}(\mathbf{r}, t)$ and $T(\mathbf{r}, t)$ are conducted with varying Ra and spatial positions across the entire cell. The mean temperature of the bulk fluid is kept at $T_{0} \simeq 30{ }^{\circ} \mathrm{C}$ and thus the Prandtl number is fixed at $\operatorname{Pr}=\nu / \kappa \simeq 5.4$.

In a recent experiment [6] we showed that the local heat transport in turbulent convection over the parameter range studied here is carried out primarily by thermal plumes, which erupt irregularly from the upper and lower thermal boundary layers. The thermal plumes organize themselves in the closed cell in such a way that the rising warm plumes accumulate on one side of the cell and the falling cold plumes concentrate on the opposite side of the cell. The spatial separation of warm and cold plumes and the resulting large-scale circulation provide a fast channel along the cell periphery for the transport of heat. Here we focus on the statistical properties of the measured $j_{z}(\mathbf{r}, t)$ at the midheight of the cell and $9 \mathrm{~mm}$ away from the sidewall. This is the plume-dominated sidewall region, in which the measured horizontal profile of the mean vertical heat flux reaches maximum [6].

Figure 1(a) shows the measured histograms of the vertical flux $P\left(j_{z}\right)$ (triangles) and horizontal flux $P\left(j_{y}\right)$ (circles). It is seen that fluctuations of the horizontal flux $j_{y}$ are symmetric and their mean value is approximately zero. Fluctuations of the vertical flux $j_{z}$, on the other hand, are highly asymmetric with positive fluctuations being much larger than negative ones. In calculating $j_{z}(\mathbf{r}, t)$, we have used the convention that warm fluctuations $\left(\delta T_{0}>0\right)$ produce positive flux if their velocities are in the upward direction $\left(v_{z}>0\right)$. With this definition, both the rising warm plumes and the falling cold plumes contribute to a positive flux. From Fig. 1(a) and the recent temperature-velocity cross-correlation measurements [6], we conclude that the measured $j_{z}$ contains both the correlated (active) and uncorrelated (passive) fluctuations. The

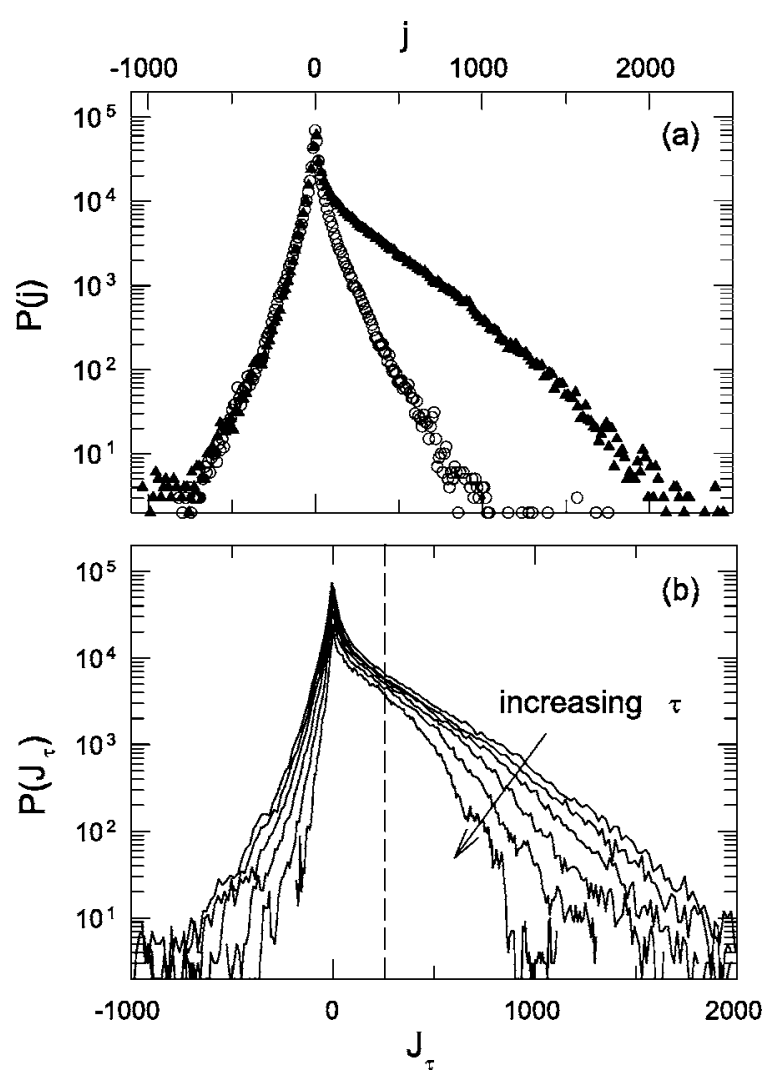

FIG. 1. (a) Comparison between the vertical flux histogram $P\left(j_{z}\right)$ (triangles) and the horizontal flux histogram $P\left(j_{y}\right)$ (circles). (b) Histograms $P\left(J_{\tau}\right)$ for $\tau=0,0.5,1,2,4,8 \mathrm{~s}$. The vertical dashed line indicates the mean value of $J_{\tau}$. All measurements are made near the sidewall at $\mathrm{Ra}=3.6 \times 10^{9}$.

negative fluctuations together with their symmetric counterpart of small positive fluctuations are produced by uncorrelated temperature and velocity signals. These fluctuations are approximately the same as those in the horizontal direction and do not contribute to the local heat transport. The correlated temperature and velocity signals are generated by the thermal plumes and produce large positive fluctuations of $j_{z}$, which give rise to a large mean value of the vertical heat flux. Put in other words, the large positive fluctuations in $j_{z}$ are generated by buoyancy, which drives convective turbulence. Small negative fluctuations in $j_{z}$ are produced by turbulent background and they are not necessarily against gravity. Figure 1(a) shows that some rare fluctuations produced by the energetic plumes (or plume clusters) can carry an instantaneous heat flux as large as 2000 . This value is more than 20 times larger than the average heating flux $(\simeq 90)$ at this Ra.

Figure 1(b) shows the histograms $P\left(J_{\tau}\right)$ of the obtained $J_{\tau}(\mathbf{r}, t)$ for six different values of $\tau$. The shape of the obtained $P\left(J_{\tau}\right)$ is highly non-Gaussian and changes continuously with increasing $\tau$. The most probable value of $J_{\tau}$ (the peak position), however, remains unchanged $(\simeq 0)$. The vertical dashed line in Fig. 1(b) indicates the mean value of $J_{\tau}$, which does not change with $\tau$ and is equal to the mean value of $j_{z}$. As the averaging time $\tau$ increases, the distribution width of $P\left(J_{\tau}\right)$ becomes narrower. This is especially evident 


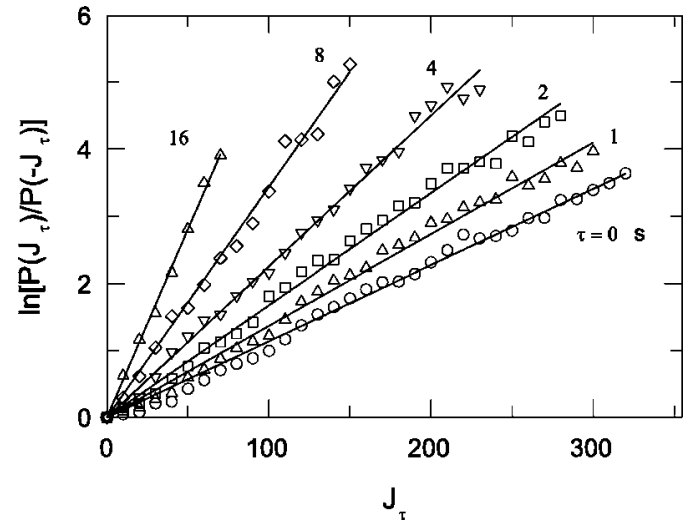

FIG. 2. Plots of $\ln \left[P\left(J_{\tau}\right) / P\left(-J_{\tau}\right)\right]$ as a function of $J_{\tau}$ at Ra $=3.6 \times 10^{9}$ for $\tau=0,1,2,4,8,16 \mathrm{~s}$. The solid lines are the linear fits to the corresponding data points.

for the negative tail part of $P\left(J_{\tau}\right)$, which determines the range of $J_{\tau}$ over which $\ln \left[P\left(J_{\tau}\right) / P\left(-J_{\tau}\right)\right]$ can be plotted. By comparing the time series $J_{\tau}(\mathbf{r}, t)$ with $j_{z}(\mathbf{r}, t)$, we find that the main effect of the time average is to filter out the fast uncorrelated fluctuations in $j_{z}(\mathbf{r}, t)$. As mentioned above, these uncorrelated fluctuations are produced mainly by the turbulent background and their probability distribution is symmetric. The large positive fluctuations in $j_{z}(\mathbf{r}, t)$ are produced by the thermal plumes. They are slow and thus remain in $J_{\tau}(\mathbf{r}, t)$.

Figure 2 shows the plots of $\ln \left[P\left(J_{\tau}\right) / P\left(-J_{\tau}\right)\right]$ as a function of $J_{\tau}$ for six different values of $\tau$. All the plots of $\ln \left[P\left(J_{\tau}\right) / P\left(-J_{\tau}\right)\right]$ are linear functions of $J_{z}$ with zero intercept, indicating that $P\left(J_{\tau}\right) / P\left(-J_{\tau}\right)$ is indeed an exponential function of $J_{\tau}$, as predicted by Eq. (3). Figure 2 also reveals that the slope $\alpha(\tau)$ of the linear fits (solid lines) increases with $\tau$.

Figure 3 shows the plots of $\ln \left[P\left(J_{\tau}\right) / P\left(-J_{\tau}\right)\right]$ as a function of $J_{\tau}$ for five different values of Ra studied in the experiment. Plots of $\ln \left[P\left(J_{\tau}\right) / P\left(-J_{\tau}\right)\right]$ obtained at different values of Ra are all linear functions of $J_{z}$ (solid lines) and the slope $\alpha(\tau)$ at

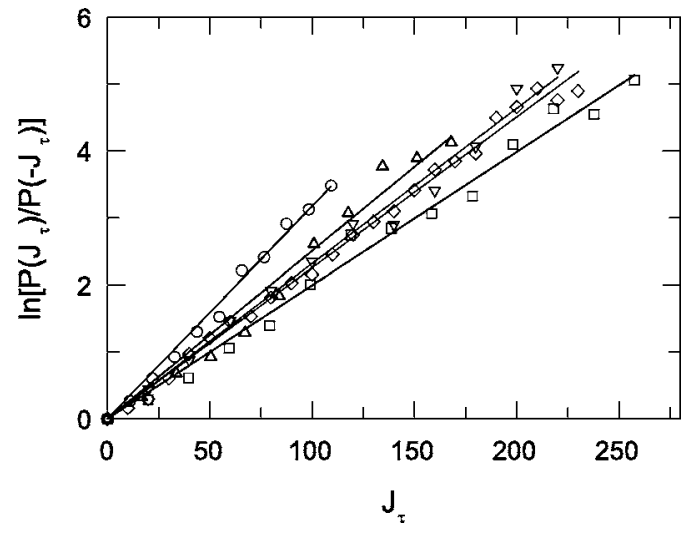

FIG. 3. Plots of $\ln \left[P\left(J_{\tau}\right) / P\left(-J_{\tau}\right)\right]$ as a function of $J_{\tau}$ with $\tau$ $=4 \mathrm{~s}$. The measurements are made at $\mathrm{Ra}=1.8 \times 10^{9}$ (circles), 2.6 $\times 10^{9}$ (up triangles), $3.6 \times 10^{9}$ (diamonds), $4.8 \times 10^{9}$ (down triangles), and $6.0 \times 10^{9}$ (squares). The solid lines show the linear fits to the corresponding data points.

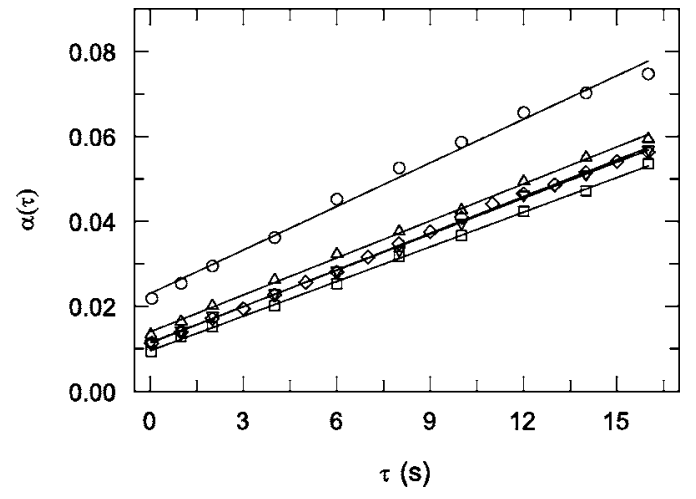

FIG. 4. Obtained slope $\alpha(\tau)$ as a function of $\tau$ for $\mathrm{Ra}=1.8$ $\times 10^{9}$ (circles), $2.6 \times 10^{9}$ (up triangles), $3.6 \times 10^{9}$ (diamonds), 4.8 $\times 10^{9}$ (down triangles), and $6.0 \times 10^{9}$ (squares). The solid lines are the linear fits to the corresponding data points.

a fixed value of $\tau(=4 \mathrm{~s})$ decreases with increasing Ra. Figure 3 thus suggests that $P\left(J_{\tau}\right) / P\left(-J_{\tau}\right)$ obtained in the Ra range studied here has a common exponential form, $\exp \left[\alpha(\tau) J_{\tau}\right]$.

Figure 4 shows the $\tau$ dependence of the slope $\alpha(\tau)$ for five different values of Ra studied in the experiment. The obtained $\alpha(\tau)$ for different values of Ra can all be fitted to a linear function $\alpha(\tau)=a \tau+b$ (solid lines). The fitted values of $a$ and $b$ are given in Table I. The nonzero value of $b$ can be thought as a correction to Eq. (3) for finite values of $\tau$, because the exponential function in Eq. (3) is an asymptotic form at $\tau \rightarrow \infty$. In fact, the value of $b$ is directly related to the asymmetry of the measured $P\left(j_{z}\right)$ shown in Fig. 1(a). This asymmetry is produced by the constant heating flux (or $\Delta T$ ) applied to keep the system at steady state. If $P\left(j_{z}\right)$ is a shifted Gaussian with a nonzero mean or an asymmetric exponential decaying function, one can show that $b \sim\left\langle j_{z}\right\rangle / \gamma_{z}^{2}$, where $\left\langle j_{z}\right\rangle$ is the local average value of $j_{z}$ and $\gamma_{z}$ is its rms value. For symmetric distribution functions, one has $\left\langle j_{z}\right\rangle=0$ and thus $b=0$. Note that the measured $P\left(j_{z}\right) / P\left(-j_{z}\right)$ at $\tau \simeq 0$ still has the exponential form.

The predicted exponential form for the probability ratio $P(+x) / P(-x)$ was also observed for other fluctuating quantities in turbulent flows $[11,12]$ and in granular flows, in which the conditions essential for the proof of the SSFT do not occur [10]. This fact suggests that the functional form of $P(+x) / P(-x)$ alone is not a sensitive test of the SSFT. Be-

TABLE I. Fitted values of $a$ and $b$ from the slope $\alpha(\tau)=a \tau+b$ obtained near the sidewall at different Ra. The numbers in the parentheses are obtained at the cell center.

\begin{tabular}{ccc}
\hline \hline $\begin{array}{c}\text { Ra } \\
\text { units of }\left(10^{9}\right)\end{array}$ & $\begin{array}{c}a \\
\left(10^{-3} \mathrm{~s}^{-1}\right)\end{array}$ & $\begin{array}{c}b \\
\text { units of }\left(10^{-2}\right)\end{array}$ \\
\hline 1.8 & $3.4(10.8)$ & $2.3(4.2)$ \\
2.6 & $2.9(7.7)$ & $1.4(3.1)$ \\
3.6 & $2.9(8.2)$ & $1.1(2.5)$ \\
4.8 & $2.8(8.1)$ & $1.1(2.4)$ \\
6.0 & $2.7(6.8)$ & $1.0(2.2)$ \\
\hline \hline
\end{tabular}


sides the functional form, the SSFT also gives a unique argument for the exponential function without any adjustable parameter. With the accurate determination of all the numerical factors involved in the experiment, one can test the SSFT more stringently. According to Eq. (3), we have $a$ $=v_{0} j_{c} \nabla_{z}(1 / T) / k_{B}$. As mentioned above, the thermal plumes that carry the local heat flux in turbulent convection are generated near the thermal boundary layers, in which the temperature gradient (and hence the thermodynamic driving force) is concentrated. The bulk fluid, on the other hand, remains isothermal on average, and temperature fluctuations in the region are not the driving force for the thermal plumes. Therefore, we have $\nabla_{z}(1 / T) \simeq \Delta T /\left(2 \lambda T_{0}^{2}\right)$ and thus $a$ $\simeq v_{0} \sigma_{c} \mathrm{Nu}$. Here $\lambda$ is the thermal boundary layer thickness, $T_{0}$ is the bulk fluid temperature, $\mathrm{Nu} \simeq H /(2 \lambda)$ is the Nusselt number (normalized total heat flux), and $\sigma_{c}=j_{c} \Delta T /\left(k_{B} T_{0}^{2} H\right)$ is an equivalent entropy production rate for conduction. At $\mathrm{Ra}=3.6 \times 10^{9}$, we have $\Delta T=17.1 \mathrm{~K}, T_{0} \simeq 303 \mathrm{~K}, \mathrm{Nu} \simeq 90$, $v_{0} \simeq 1 \mathrm{~mm}^{3}, \quad k=0.615 \mathrm{~W} / \mathrm{K} \mathrm{m}$ and thus $\sigma_{c} \simeq 3.4$ $\times 10^{15} \mathrm{~cm}^{3} \mathrm{~s}^{-1}$ and $a \simeq 3 \times 10^{14} \mathrm{~s}^{-1}$. The calculated value of $a$ is $10^{17}$ times larger than the fitted value.

The above results suggest that the SSFT can indeed be extended to the local variables, but there are several important issues remained to be further clarified. First, to balance Eq. (3) one needs to introduce an effective temperature $T_{\text {eff }}$ $\simeq 3.2 \times 10^{8} T_{0}$ or an energy scale $k_{B} T_{\text {eff }} \simeq 10^{-12} \mathrm{~J}$. This energy is comparable to the turbulent kinetic energy $E_{k}$ $=(1 / 2) \rho \lambda^{3} v_{r m s}^{2}$, where $\rho=1 \mathrm{~g} / \mathrm{cm}^{3}$ is the fluid density, $v_{r m s}$ $\simeq 0.4 \mathrm{~cm} / \mathrm{s}$ [13] is the rms velocity, and $\lambda \simeq 0.8 \mathrm{~mm}$ [6] is the smallest length scale in turbulent convection. In fact, the dynamic equations for turbulent convection are independent of $T_{0}$ (except that some fluid parameters may vary with $T_{0}$ ). A proper definition of $T_{e f f}$ in terms of measurable dynamic variables is needed for systems far from equilibrium $[9,10,14]$. Without such a definition, Eq. (1) will not remain free of adjustable parameters as it appears to be. Second, we find that the fitted value of $a$ changes with the spatial position in the convection cell. As shown in Table I, the value of $a$ obtained at the cell center is $\sim 2.8$ times larger than that near the sidewall. This is caused by the nonuniform distribution of thermal plumes in the closed cell. In fact, large-scale coherent structures are often found in systems far from equilibrium and they have a profound influence on fluctuations of the local variables $[1,4,5]$.

In summary, the experiment clearly reveals that the probability ratio $P\left(+J_{\tau}\right) / P\left(-J_{\tau}\right)$ in turbulent thermal convection has a common exponential form $\exp \left[a J_{\tau} \tau\right]$, as predicted by the SSFT. Systematic measurements of the local entropy production rate $\sigma(\mathbf{r}, t)$ over varying $\mathrm{Ra}$ and spatial positions in the cell show that fluctuations of the time-averaged $\sigma(\mathbf{r}, t)$ are neither isotropic nor homogeneous. The amplitude of the fluctuations is determined by an energy scale much larger than $k_{B} T$ but comparable to the turbulent kinetic energy at the smallest length scale of the convective flow. How to incorporate these common dynamic complexities into the SSFT for the local variables is certainly a challenge for theory. Our results together with the early measurements [10-12] suggest that the SSFT may be further extended to include other fluctuating variables in turbulent flows and in granular flows.

We thank W. I. Goldburg and X.-R. Wang for useful discussions. This work was supported in part by the NSF under Grant No. DMR-0071323 (P.T.) and by the RGC of Hong Kong SAR under Grant Nos. HKUST603003 (P.T.) and CUHK403003 (K.Q.X.).
[1] D. Ruelle, Phys. Today 57(5), 48 (2004).

[2] D. J. Evans and D. J. Searles, Adv. Phys. 51, 1529 (2002), and references therein.

[3] G. Gallavotti, Physica A 263, 39 (1999).

[4] L. P. Kadanoff, Phys. Today 54(8), 34 (2001).

[5] S. Grossmann and D. Lohse, J. Fluid Mech. 407, 27 (2000).

[6] X.-D. Shang et al., Phys. Rev. Lett. 90, 074501 (2003); Phys. Rev. E 70, 026308 (2004).

[7] D. Kondepudi and I. Prigogine, Modern Thermodynamics (Wiley, New York, 1998), p. 385.
[8] G. Gallavotti and E. G. D. Cohen, Phys. Rev. Lett. 74, 2694 (1995); J. Stat. Phys. 80, 931 (1995).

[9] W. I. Goldburg et al., Phys. Rev. Lett. 87, 245502 (2001); T. Toth-Katona et al., Phys. Rev. E 68, 030101(R) (2003).

[10] K. Feitosa and N. Menon, Phys. Rev. Lett. 92, 164301 (2004).

[11] S. Ciliberto and C. Laroche, J. Phys. IV 8, 215 (1998).

[12] S. Ciliberto et al., Physica A 340, 240 (2004).

[13] X.-L. Qiu and P. Tong, Phys. Rev. E 64, 036304 (2001).

[14] G. Gallavotti and E. G. D. Cohen, e-print cond-mat/0312306. 